\title{
On $(2,2)$-regular Non-associative Ordered Semigroups via Its Semilattices and Generated (Generalized Fuzzy) Ideals
}

\author{
Yousef Al-Qudah $^{1, *}$, Faisal Yousafzai ${ }^{2}$, Mohammed M. Khalaf ${ }^{3}$, Mohammad Almousa ${ }^{1}$ \\ ${ }^{1}$ Department of Mathematics, Faculty of Arts and Science, Amman Arab University, Amman, Jordan \\ ${ }^{2}$ Military College of Engineering, National University of Sciences and Technology (NUST), Islamabad, Pakistan \\ ${ }^{3}$ Higher Institute of Engineering and Technology, King Mariout, Egypt
}

Received February 8, 2020; Revised April 23, 2020; Accepted May 3, 2020

Copyright $@ 2020$ by authors, all rights reserved. Authors agree that this article remains permanently open access under the terms of the Creative Commons Attribution License 4.0 International License

\begin{abstract}
The main motivation behind this paper is to study some structural properties of a non-associative structure as it hasn't attracted much attention compared to associative structures. In this paper, we introduce the concept of an ordered $\mathcal{A}^{*} \mathcal{G}^{* *}$-groupoid and provide that this class is more generalized than an ordered $\mathcal{A G}$-groupoid with left identity. We also define the generated left (right) ideals in an ordered $\mathcal{A}^{*} \mathcal{G}^{* *}$-groupoid and characterize a $(2,2)$-regular ordered $\mathcal{A}^{*} \mathcal{G}^{* *}$-groupoid in terms of these ideals. We then study the structural properties of an ordered $\mathcal{A}^{*} \mathcal{G}^{* *}$-groupoid in terms of its semilattices, $(2,2)$-regular class and generated commutative monoids. Subsequently, compare $\left(\epsilon_{\gamma}, \in_{\gamma} \vee q_{\delta}\right)$-fuzzy left/right ideals of an ordered AG-groupoid and respective examples are provided. Relations between an $\left(\epsilon_{\gamma}, \in_{\gamma} \vee q_{\delta}\right)$-fuzzy idempotent subsets of an ordered $\mathrm{A}^{*} \mathrm{G}^{* *}$-groupoid and its $\left(\epsilon_{\gamma}, \in_{\gamma} \vee q_{\delta}\right)$-fuzzy bi-ideals are discussed. As an application of our results, we get characterizations of $(2,2)$-regular ordered $\mathcal{A}^{*} \mathcal{G}^{* *}$-groupoid in terms of semilattices and $\left(\epsilon_{\gamma}, \epsilon_{\gamma} \vee q_{\delta}\right)$-fuzzy left (right) ideals. These concepts will help in verifying the existing characterizations and will help in achieving new and generalized results in future works.
\end{abstract}

Keywords Ordered $\mathcal{A G}$-groupoid, Non-associativity, Ordered $\mathcal{A}^{*} \mathcal{G}^{* *}$-groupoid, Left Invertive Law, Generated Ideals and $\left(\in_{\gamma}, \in_{\gamma} \vee q_{\delta}\right)$-fuzzy-ideals.

\section{Introduction}

An $\mathcal{A G}$-groupoid can be referred to as a non-associative semigroup, as the main difference between semigroups and $\mathcal{A G}$-groupoids is the switching of an associative law. An $\mathcal{A G}$ groupoid is a non-associative and a non-commutative algebraic structure lying in a grey area between a groupoid and a com- mutative semigroup. Commutative law is given by $a b c=c b a$ in ternary operations. By putting brackets on the left of this equation, i.e. $(a b) c=(c b) a$, in 1972, Kazim and Naseeruddin introduced a new algebraic structure called a left almost semigroup abbreviated as an $\mathcal{L} \mathcal{A}$-semigroup [1]. This identity is called the left invertive law. Protic and Stevanovic called the same structure an Abel-Grassmann's groupoid abbreviated as an AG-groupoid [2].

This structure is closely related to a commutative semigroup because an $\mathcal{A G}$-groupoid with right identity becomes a commutative semigroup [3]. It was proved in [1] that an $\mathcal{A G}$ groupoid $S$ is medial, that is, $a b \cdot c d=a c \cdot b d$ holds for all $a, b, c, d \in S$. An $\mathcal{A} \mathcal{G}$-groupoid may or may not contain a left identity. The left identity of an $\mathcal{A} \mathcal{G}$-groupoid permits the inverses of elements in the structure. If an $\mathcal{A G}$-groupoid contains a left identity, then this left identity is unique [3]. In an $\mathcal{A G}$-groupoid $S$ with left identity (unitary $\mathcal{A G}$-groupoid), the paramedial law $a b \cdot c d=d c \cdot b a$ holds for all $a, b, c, d \in S$. By using medial law with left identity, we get $a \cdot b c=b \cdot a c$ for all $a, b, c \in S$. We should genuinely acknowledge that much of the ground work has been done by Kazim, Naseeruddin, Mushtaq, Kamran, Protic, Stevanovic, Khan, Dudek and Gigon. One can be referred to $[2,3,4,5,6,7,8]$ in this regard.

\section{Preliminaries}

The concept of fuzzy sets was first proposed by Zadeh [9] in 1965 , which has a wide range of applications in various fields such as computer engineering, artificial intelligence, control engineering, operation research, management science, robotics and many more. It gives us a tool to model the uncertainty present in a phenomena that does not have sharp boundaries. After Zadeh's work, interesting results of fuzzy set theory have been proposed, such as complex fuzzy sets $[10,11,12]$, algebra $[15,16]$, real analysis, measure theory and topology etc. 
Murali [13] defined the concept of belongingness of a fuzzy point to a fuzzy subset under a natural equivalence on a fuzzy subset. In [14], the idea of quasi-coincidence of a fuzzy point with a fuzzy set is defined. A new type of fuzzy subgroup, that is $(\alpha, \beta)$-fuzzy subgroup, was introduced in an earlier paper of Bhakat and Das [15] by using the notions of "belongingness and quasi-coincidence" of fuzzy points and fuzzy sets. The concepts of an $(\in, \in \vee q)$-fuzzy subgroup is a useful generalization of Rosenfeld's fuzzy subgroups [16]. It is now natural to investigate similar type of generalizations of existing fuzzy sub-systems of other algebraic structures. The concept of an $(\in, \in \vee q)$-fuzzy sub-near rings of a near ring was introduced by Davvaz in [17]. In [18], Kazanchi and Yamak studied $(\in, \in \vee q)$-fuzzy bi-ideals of a semigroup. In [19], Shabir et. al. characterized regular semigroups by the properties of $(\in, \in \vee q)$-fuzzy ideals, fuzzy bi-ideals and fuzzy quasi-ideals. In [18], Kazanchi and Yamak defined $(\bar{\epsilon}, \bar{\in} \vee \bar{q})$-fuzzy bi-ideals in semigroups. Many other researchers used the idea of generalized fuzzy sets and gave several characterizations results in different branches of algebra. Generalizing the concept of $x_{t} q f$, Shabir and Jun [20], defined $x_{t} q_{k} f$ as $f(x)+t+k>1$, where $k \in[0,1)$. In [20], semigroups are characterized by the properties of their $\left(\in, \in \vee q_{k}\right)$-fuzzy ideals.

In the present paper, we introduce and investigate the notions of $\left(\epsilon_{\gamma}, \epsilon_{\gamma} \vee q_{\delta}\right)$-fuzzy left (right) ideals and $\left(\epsilon_{\gamma}, \in_{\gamma} \vee q_{\delta}\right)$ fuzzy bi-ideals, and study the relationship between these ideals in detail. As an application of our results we get characterizations of a $(2,2)$-regular class of an ordered $\mathcal{A}^{*} \mathcal{G}^{* *}$-groupoid in terms of its semilattices, one-sided (two-sided) ideals and bi-ideals based on fuzzy sets and its associated fuzzy points.

The concept of an ordered $\mathcal{A G}$-groupoid was given by Yousafzai et. al. in [21] as follows:

An $\mathcal{A G}$-groupoid $(S, \cdot)$ together with a partial order $\leq$ on $S$ that is compatible with an $\mathcal{A G}$-groupoid operation, meaning that for $x, y, z \in S, x \leq y \Rightarrow z x \leq z y$ and $x z \leq y z$, is called an ordered $\mathcal{A G}$-groupoid [21].

Note that an ordered $\mathcal{A G}$-groupoid is the generalization of an ordered semigroup because if an ordered $\mathcal{A G}$-groupoid has a right identity then it becomes an ordered semigroup.

- Let $\emptyset \neq A \subseteq S$, we denote $(A]$ by $(A]:=\{x \in S / x \leq a$ for some $a \in A\}$. If $A=\{a\}$, then we write $(\{a\}]$. $B\}$.

- For $\emptyset \neq A, B \subseteq S$, we denote $A B=:\{a b / a \in A, b \in$

- A nonempty subset $A$ of an ordered $\mathcal{A G}$-groupoid $S$ is called a left (right) ideal of $S$ if:

(i) $S A \subseteq A(A S \subseteq A)$;

(ii) if $a \in A$ and $b \in S$ such that $b \leq a$, then $b \in A$.

- By two-sided ideal or simply ideal, we mean a nonempty subset of an ordered $\mathcal{A G}$-groupoid $S$ which is both left and right ideal of $S$.

- A nonempty subset $A$ of an ordered $\mathcal{A G}$-groupoid $S$ is called an $\mathcal{A G}$-subgroupoid of $S$ if $A^{2} \subseteq A$.

- An $\mathcal{A G}$-subgroupoid $A$ of an ordered $\mathcal{A G}$-groupoid $S$ is called a bi-ideal of $S$ if:

(i) $A \cdot S A \subseteq A$;

(ii) if $a \in A$ and $b \in S$ such that $b \leq a$, then $b \in A$.

Equivalently: A nonempty subset $A$ of an ordered $\mathcal{A G}$ groupoid $S$ is called a left (right) ideal of $S$ if $(A \cdot S A] \subseteq A$.
A fuzzy subset $f$ of a given set $S$ is described as an arbitrary function $f: S \longrightarrow[0,1]$, where $[0,1]$ is the usual closed interval of real numbers [9]. For any two fuzzy subsets $f$ and $g$ of $S, f \subseteq g$ means that, $f(x) \leq g(x), \forall x \in S$.

Let $f$ and $g$ be any fuzzy subsets of an ordered $\mathcal{A G}$-groupoid $S$, then the product $f \circ g$ is defined by

$(f \circ g)(a)$

$=\left\{\begin{array}{lr}\bigvee_{a \leq b c}\{f(b) \wedge g(c)\}, & \text { if } \exists b, c \in S, \text { such that } a \leq b c \\ 0, & \text { otherwise. }\end{array}\right.$

- Let $\mathcal{F}(S)$ denotes the collection of all fuzzy subsets of an ordered $\mathcal{A G}$-groupoid $S$, then it is easy to see that $(\mathcal{F}(S), \circ, \subseteq)$ becomes an ordered $\mathcal{A} \mathcal{G}$-groupoid [21].

- The characteristic function $\mathcal{X}_{A}$ for a non-empty $A$ of an ordered $\mathcal{A G}$-groupoid $S$ is defined by

$$
\mathcal{X}_{A}(x)=\left\{\begin{array}{l}
1, \text { if } x \in A \\
0, \text { if } x \notin A
\end{array}\right.
$$

- A fuzzy subset $f$ of an ordered $\mathcal{A G}$-groupoid $S$ of the form

$$
f(y)=\left\{\begin{array}{c}
r(\neq 0), \text { if } y \leq x \\
0, \quad \text { otherwise }
\end{array}\right.
$$

is said to be a fuzzy point with support $x$ and value $r$ and is denoted by $x_{r}$, where $r \in(0,1]$.

- In what follows let $\gamma, \delta \in[0,1]$ be such that $\gamma<\delta$. For any $B \subseteq A$, we define $\mathcal{X}_{\gamma B}^{\delta}$ be the fuzzy subset of $S$ by $\mathcal{X}_{\gamma B}^{\delta}(x) \geq \delta$ and $\mathcal{X}_{\gamma B}^{\delta}(x) \leq \gamma, \forall x \in B$. Otherwise, clearly $\mathcal{X}_{\gamma B}^{\delta}$ is the characteristic function of $B$ if $\gamma=0$ and $\delta=1$.

- For a fuzzy point $x_{r}$ and a fuzzy subset $f$ of an ordered $\mathcal{A G}$-groupoid $S$, we say that:

(i) $x_{r} \in_{\gamma} f$ if $f(x) \geq r>\gamma$.

(ii) $x_{r} q_{\delta} f$ if $f(x)+r>2 \delta$.

(iii) $x_{r} \in_{\gamma} \vee q_{\delta} f$ if $x_{r} \in_{\gamma} f$ or $x_{r} q_{\delta} f$.

- Now we introduce a new relation on $\mathcal{F}(S)$, denoted as " $\subseteq \vee q_{(\gamma, \delta)}$ ", as follows.

For any $f, g \in \mathcal{F}(S)$, by $f \subseteq \vee q_{(\gamma, \delta)} g$, we mean that $x_{r} \in_{\gamma} f \Longrightarrow x_{r} \in_{\gamma} \vee q_{\delta} g, \forall x \in S$ and $r \in(\gamma, 1]$. Moreover $f$ and $g$ are said to be $(\gamma, \delta)$-equal, denoted by $f={ }_{(\gamma, \delta)} g$, if $f \subseteq \vee q_{(\gamma, \delta)} g$ and $g \subseteq \vee q_{(\gamma, \delta)} f$.

Lemma 1. [22] Let $f, g, h \subseteq \mathcal{F}(S)$ and $\gamma, \delta \in[0,1]$, then the following conditions hold:

(i) $f \subseteq \vee q_{(\gamma, \delta)} g\left(f \supseteq \vee q_{(\gamma, \delta)} g\right) \Leftrightarrow \max \{f(x), \gamma\} \leq$ $\min \{g(x), \delta\}(\max \{f(x), \gamma\} \geq \min \{g(x), \delta\}), \forall x \in S$.

(ii) If $f \subseteq \vee q_{(\gamma, \delta)} g$ and $g \subseteq \vee q_{(\gamma, \delta)} h$, then $f \subseteq \vee q_{(\gamma, \delta)} h$.

Corollary 1. $=\vee q_{(\gamma, \delta)}$ is an equivalence relation on $\mathcal{F}(S)$.

- By Lemma 1, it is also notified that $f=\vee q_{(\gamma, \delta)} g \Leftrightarrow$ $\max \{\min \{f(x), \delta\}, \gamma\}=\max \{\min \{g(x), \delta\}, \gamma\}, \forall x \in S$, where $\gamma, \delta \in[0,1]$.

Lemma 2. [22] Let $A$ and $B$ be any subsets of an ordered $\mathcal{A G}$-groupoid $S$, where $r \in(\gamma, 1]$ and $\gamma, \delta \in[0,1]$, then: 
(1) $A \subseteq B \Leftrightarrow \mathcal{X}_{\gamma A}^{\delta} \subseteq \vee q_{(\gamma, \delta)} \mathcal{X}_{\gamma B}^{\delta}$;

$(2) \mathcal{X}_{\gamma A}^{\delta} \cap \mathcal{X}_{\gamma B}^{\delta}={ }_{(\gamma, \delta)} \mathcal{X}_{\gamma(A \cap B)}^{\delta}$;

(3) $\mathcal{X}_{\gamma A}^{\delta} \circ \mathcal{X}_{\gamma B}^{\delta}={ }_{(\gamma, \delta)} \mathcal{X}_{\gamma(A B]}^{\delta}$.

- A fuzzy subset $f$ of an ordered $\mathcal{A G}$-groupoid $S$ is called an $\left(\in_{\gamma}, \in_{\gamma} \vee q_{\delta}\right)$-fuzzy $\mathcal{A} \mathcal{G}$-subgroupoid of $S$ if for all $a, b \in S$ and $s, t \in(\gamma, 1]$, the following conditions hold:

(i) If $a \leq b$ and $b_{t} \in_{\gamma} f \Longrightarrow a_{t} \in_{\gamma} \vee q_{\delta} f$;

(ii) If $a_{t} \in_{\gamma} f$ and $b_{t} \in_{\gamma} f \Longrightarrow(a b)_{\min \{t, s\}} \in_{\gamma} \vee q_{\delta} f$.

- A fuzzy subset $f$ of an ordered $\mathcal{A G}$-groupoid $S$ is called an $\left(\in_{\gamma}, \in_{\gamma} \vee q_{\delta}\right)$-fuzzy left (right) ideal of $S$ if for all $a, b \in S$ and $t \in(\gamma, 1]$, the following conditions hold:

(i) If $a \leq b$ and $b_{t} \in_{\gamma} f \Longrightarrow a_{t} \in_{\gamma} \vee q_{\delta} f$;

(ii) If $b_{t} \in_{\gamma} f \Longrightarrow(a b)_{t} \in_{\gamma} \vee q_{\delta} f\left(a_{t} \in_{\gamma} f \Longrightarrow(a b)_{t} \in_{\gamma}\right.$ $\left.\vee q_{\delta} f\right)$.

- A fuzzy subset $f$ of an ordered $\mathcal{A G}$-groupoid $S$ is called an $\left(\in_{\gamma}, \in_{\gamma} \vee q_{\delta}\right)$-fuzzy bi-ideal of $S$ if for all $x, y, z \in S$ and $s, t \in(\gamma, 1]$, the following conditions hold:

(i) If $a \leq b$ and $b_{t} \in_{\gamma} f \Longrightarrow a_{t} \in_{\gamma} \vee q_{\delta} f$;

(ii) if $x_{t} \in_{\gamma} f$ and $y_{s} \in_{\gamma} f \Longrightarrow(x y)_{\min \{t, s\}} \in_{\gamma} \vee q_{\delta} f$;

(iii) if $x_{t} \in_{\gamma} f$ and $z_{s} \in_{\gamma} f \Longrightarrow((x y) z)_{\min \{t, s\}} \in_{\gamma} \vee q_{\delta} f$.

The following results can be find in [22].

Theorem 1. A fuzzy subset $f$ of an ordered $\mathcal{A G}$-groupoid $S$ is called an $\left(\in_{\gamma}, \in_{\gamma} \vee q_{\delta}\right)$-fuzzy left (right) ideal of $S$ if for all $a, b \in S$ and $\gamma, \delta \in[0,1]$, the following conditions hold:

(i) $\max \{f(a), \gamma\} \geq \min \{f(b), \delta\}$ with $a \leq b$;

(ii) $\max \{f(a b), \gamma\} \geq \min \{f(b), \delta\}$.

Theorem 2. A fuzzy subset $f$ of an ordered $\mathcal{A G}$-groupoid $S$ is called an $\left(\epsilon_{\gamma}, \in_{\gamma} \vee q_{\delta}\right)$-fuzzy $\mathcal{A G}$-subgroupoid if for all $a, b \in S$ and $t \in(\gamma, 1]$, the following conditions hold:

(i) $\max \{f(a), \gamma\} \geq \min \{f(b), \delta\}$ with $a \leq b$;

(ii) $\max \{f(a b), \gamma\} \geq \min \{f(a), f(b), \delta\}$.

Theorem 3. A fuzzy subset $f$ of an ordered $\mathcal{A G}$-groupoid $S$ is called an $\left(\epsilon_{\gamma}, \in_{\gamma} \vee q_{\delta}\right)$-fuzzy bi-ideal of $S$ if for all $x, y, z \in$ $S, s, t \in(\gamma, 1]$ and $\gamma, \delta \in[0,1]$, the following conditions hold:

(i) $\max \{f(a), \gamma\} \geq \min \{f(b), \delta\}$ with $a \leq b$;

(ii) $\max \{f(x y), \gamma\} \geq \min \{f(x), f(y), \delta\}$;

(iii) $\max \{f((x y) z), \gamma\} \geq \min \{f(x), f(z), \delta\}$.

Lemma 3. Let $f$ be a fuzzy subset of an ordered $\mathcal{A G}$ groupoid $S$ and $\gamma, \delta \in[0,1]$, then $f$ is an $\left(\epsilon_{\gamma}, \in_{\gamma} \vee q_{\delta}\right)$-fuzzy left (right, bi-) ideal of $S$ if and only if $f$ satisfies the following conditions.

(i) $x \leq y \Rightarrow \max \{f(x), \gamma\} \geq \min \{g(x), \delta\}, \forall x, y \in S$;

(ii) $S \circ f \subseteq \vee q_{(\gamma, \delta)} f\left(f \circ S \subseteq \vee q_{(\gamma, \delta)} f\right.$, $\left.(f \circ S) \circ f \subseteq \vee q_{(\gamma, \delta)} f\right)$.

Lemma 4. Let $A$ be a non-empty subset of an ordered $\mathcal{A G}$-groupoid $S$, then $A$ is a left (right, bi-) ideal of $S \Leftrightarrow \mathcal{X}_{\gamma A}^{\delta}$ is an $\left(\epsilon_{\gamma}, \in_{\gamma} \vee q_{\delta}\right)$-fuzzy left (right, bi-) ideal of $S$, where $\gamma, \delta \in[0,1]$.
Remark 1. If $S$ is an ordered $\mathcal{A G}$-groupoid, then $S \circ S=S$.

Example 1. Let $S=\{a, b, c\}$ be an ordered $\mathcal{A} \mathcal{G}$-groupoid with the following multiplication table and two different orders below :

$$
\begin{aligned}
& \begin{array}{l|lll}
\cdot & a & b & c \\
\hline a & a & a & a \\
b & a & a & c \\
c & a & a & a
\end{array} \\
& \leq:=\{(a, a),(b, b),(c, c),(c, a),(c, b)\} \\
& \leq:=\{(a, a),(b, b),(c, c),(a, c),(a, b)\}
\end{aligned}
$$

Let us consider an Example 1 of an ordered $\mathcal{A G}$-groupoid with order (2). Let $\gamma=0.4$ and $\delta=0.5$. Define a fuzzy subset $f: S \rightarrow[0,1]$ as follows:

$$
f(x)=\left\{\begin{array}{l}
0.7 \text { for } x=a \\
0.8 \text { for } x=b \\
0.9 \text { for } x=c
\end{array}\right.
$$

(1) Let us consider all the possible cases for $t \in(0.4,1]$ as follows:

(i) When $t \in(0.4,0.7]$, then $x_{t} \in \in_{\gamma} f$ for all $x \in S$. It is easy to see that $x_{t} \in_{\gamma} f$ and $y \leq x \Longrightarrow y_{t} \in_{\gamma} f$ for all $x \in S$.

(ii) When $t \in(0.7,0.8]$, then $a_{t} \bar{\epsilon}_{\gamma} f$ while $c_{t} \in_{\gamma} f$ and $b_{t} \in_{\gamma} f$. Now $a \leq c$ and $c_{t} \in_{\gamma} f \Longrightarrow f(a) \geq t>\gamma$. Proceeding in the same way as in above example we get $a_{t} q_{\delta} f$, and similar solution for $a \leq b$.

(iii) When $t \in(0.8,0.9]$, then $c_{t} \in_{\gamma} f$ while $a_{t} \bar{\epsilon}_{\gamma} f$ and $b_{t} \bar{\epsilon}_{\gamma} f$. It is easy to verify that $c_{t} \in_{\gamma} f$ and $a \leq c \Longrightarrow a_{t} q_{\delta} f$.

(iv) When $t \in(0.9,1]$, then $\bar{x}_{t} \in \in_{\gamma} f$ for all $x \in S$. Nothing to show in this case.

(2) Again considering all possible cases for $t \in(0.4,1]$ as follows:

(i) When $t \in(0.4,0.7]$, then $x_{t} \in_{\gamma} f$ for all $x \in S$. It is easy see that $(x y)_{t} \in_{\gamma} f$ for all $x \in S$ in this case.

(ii) When $t \in(0.7,0.8]$, then $a_{t} \bar{\epsilon}_{\gamma} f$ while $c_{t} \in_{\gamma} f$ and $b_{t} \in_{\gamma} f$. Now $b_{t} \in_{\gamma} f \Longrightarrow(a b)_{t} q_{\delta} f,(b b)_{t} q_{\delta} f$ and $(b c)_{t} q_{\delta} f$. Similarly $c_{t} \in f \Longrightarrow(a c)_{t} q_{\delta} f,(b c)_{t} \in \in_{\gamma} f$ and $(c c)_{t} q_{\delta} f$.

(iii) When $t \in(0.8,0.9]$, then $c_{t} \in_{\gamma} f$ while $a_{t} \bar{\epsilon}_{\gamma} f$ and $b_{t} \bar{\epsilon}_{\gamma} f$. Now $c_{t} \in f \Longrightarrow(a c)_{t} q_{\delta} f,(b c)_{t} \in \in_{\gamma} f$ and $(c c)_{t} q_{\delta} f$.

(iv) When $t \in(0.9,1]$, then $\bar{x}_{t} \in_{\gamma} f$ for all $x \in S$. Again nothing to solve in this case.

Hence $f$ is an $\left(\epsilon_{\gamma}, \in_{\gamma} \vee q_{\delta}\right)$-fuzzy left ideal of $S$. Similarly we can provide an example for right ideal as well.

Again consider an example 1 of an ordered $\mathcal{A G}$-groupoid with order (1). Let $\gamma=0.45$ and $\delta=0.49$. Define a fuzzy subset $f: S \rightarrow[0,1]$ as follows:

$$
f(x)=\left\{\begin{array}{c}
0.9 \text { for } x=a \\
0.99 \text { for } x=b . \\
0.84 \text { for } x=c
\end{array}\right.
$$


(1) Let us consider all the possible cases for $t \in(0.45,1]$ as follows:

(i) When $t \in(0.45,0.84]$, then $x_{t} \in_{\gamma} f$. It is easy to see that $x_{t} \in_{\gamma} f$ and $y \leq x \Longrightarrow y_{t} \in_{\gamma} f$ for all $x \in S$.

(ii) When $t \in(0.84,0.9]$, then $c_{t} \bar{\epsilon}_{\gamma} f$ while $a_{t} \in_{\gamma} f$ and $b_{t} \in_{\gamma} f$. Now $c \leq a$ and $a_{t} \in_{\gamma} f \Longrightarrow f(a) \geq t>\gamma$. Consider $f(c)+t>0.84+0.84=1.64>2 \delta$. Hence $c \leq a$ and $a_{t} \in_{\gamma} f \Longrightarrow c_{t} q_{\delta} f$. Similarly $b_{t} \in_{\gamma} f$ and $c \leq b \Longrightarrow c_{t} q_{\delta} f$.

(iii) When $t \in(0.9,0.99]$, then $b_{t} \in_{\gamma} f$ while $a_{t} \bar{\epsilon}_{\gamma} f$ and $c_{t} \bar{\epsilon}_{\gamma} f$.It is easy to verify that $b_{t} \in_{\gamma} f$ and $c \leq b \Longrightarrow c_{t} q_{\delta} f$.

(iv) When $t \in(0.99,1]$, then $x_{t} \bar{\epsilon}_{\gamma} f$ for all $x \in S$. Hence nothing to show in this case.

(2) Now let us consider some basic comparisons as follows:

(i) $a_{t} \in_{\gamma} f, a_{s} \in_{\gamma} f \Longrightarrow(a a)_{\min \{s, t\}} \in_{\gamma} f$.

(ii) $a_{t} \in_{\gamma} f, b_{s} \in_{\gamma} f \Longrightarrow(a b)_{\min \{s, t\}} \in_{\gamma} f$.

(iii) $a_{t} \in_{\gamma} f, c_{s} \in_{\gamma} f \Longrightarrow(a c)_{\min \{s, t\}} q_{\delta} f$.

(iv) $b a=a b$ in the table.

(v) $b_{t} \in_{\gamma} f, b_{s} \in_{\gamma} f \Longrightarrow(b b)_{\min \{s, t\}} \in_{\gamma} f$.

(vi) $b_{t} \in_{\gamma} f, c_{s} \in_{\gamma} f \Longrightarrow(b c)_{\min \{s, t\}} \in_{\gamma} f$.

(vii) $c a=a c$ in the table.

(viii) $c_{t} \in_{\gamma} f, b_{s} \in_{\gamma} f \Longrightarrow(c b)_{\min \{s, t\}} \in_{\gamma} f$.

$(i x) c_{t} \in_{\gamma} f, c_{s} \in_{\gamma} f \Longrightarrow(c c)_{\min \{s, t\} .} \in_{\gamma} f$.

Hence $f$ is $\left(\in_{\gamma}, \in_{\gamma} \vee q_{\delta}\right)$-fuzzy $\mathcal{A} \mathcal{G}$-subgroupoid of $S$.

- A fuzzy subset $f$ of an ordered $\mathcal{A G}$-groupoid $S$ is called an $\left(\epsilon_{\gamma}, \in_{\gamma} \vee q_{\delta}\right)$-fuzzy semiprime if for all $a \in S$ and $\gamma, \delta \in[0,1]$, if $\max \{f(a), \gamma\} \geq \min \left\{f\left(a^{2}\right), \delta\right\}$.

Lemma 5. Let $A$ be any right (left) ideal of an ordered $\mathcal{A G}$-groupoid $S$. Then $A$ is semiprime if and only if $\mathcal{X}_{A}$ is $\left(\in_{\gamma}, \in_{\gamma} \vee q_{\delta}\right)$-fuzzy semiprime.

Proof It is simple.

\section{On $\left(\epsilon_{\gamma}, \epsilon_{\gamma} \vee q_{\delta}\right)$-fuzzy ideals of $(2,2)$ - regular ordered $\mathcal{A}^{*} \mathcal{G}^{* * *}$-groupoids}

\subsection{Basic results and examples}

This section contains some examples and basic results which will be essential for up coming section.

Example 2. Let us consider an example 1 of an ordered $\mathcal{A G}$ groupoid with order (2). Define a fuzzy subset $f: S \rightarrow[0,1]$ as follows.

$$
f(x)=\left\{\begin{array}{l}
0.9 \text { for } x=1 \\
0.6 \text { for } x=2 \\
0.7 \text { for } x=3
\end{array} .\right.
$$

Then by routine calculation it is easy to observe the following:

(i) $f$ is an $\left(\epsilon_{0.3}, \in_{0.3} \vee q_{0.4}\right)$-fuzzy two-sided ideal of $S$.

(ii) $f$ is not an $\left(\in, \in \vee q_{0.3}\right)$-fuzzy two-sided ideal of $S$, because $f(12)<f(2) \wedge \frac{1-0.3}{2}$.

Example 3. Let $S=\{w, x, y, z\}$ be an ordered $\mathcal{A G}$ groupoid define in the following multiplication table and or- dered below.

$$
\begin{array}{r|llll}
\cdot & w & x & y & z \\
\hline w & w & w & w & w \\
x & w & w & w & w \\
y & w & w & w & x \\
z & w & w & x & y \\
\leq:=\{(w, w),(x, x),(y, y),(z, z),(w, x)\}
\end{array}
$$

Define a fuzzy subset $f: S \rightarrow[0,1]$ as follows:

$$
f(x)=\left\{\begin{array}{c}
0.75 \text { for } x=w \\
0.65 \text { for } x=x \\
0.7 \text { for } x=y \\
0.5 \text { for } x=z
\end{array}\right.
$$

Then clearly $f$ is an $\left(\in_{0.3}, \in_{0.3} \vee q_{0.4}\right)$-fuzzy left ideal of $S$. Again define a fuzzy subset $f: S \rightarrow[0,1]$ as follows:

$$
f(x)=\left\{\begin{array}{l}
0.9 \text { for } x=w \\
0.7 \text { for } x=x \\
0.6 \text { for } x=y \\
0.5 \text { for } x=z
\end{array}\right.
$$

Then $f$ is an $\left(\in_{0.2}, \in_{0.2} \vee q_{0.5}\right)$-fuzzy bi-ideal.

Lemma 6. [21] Let $S$ be an ordered $\mathcal{A G}$-groupoid and $\emptyset \neq$ $A, B \subseteq S$. Then the followings hold:

(i) $A \subseteq(A]$;

(ii) If $A \subseteq B$, then $(A] \subseteq(B]$;

(iii) $(A](B] \subseteq(A B]$;

(iv) $(A]=((A]]$;

(vi) $((A])(B]]=(A B]$;

(vii) $(T]=T$, for every ideal $T$ of $S$;

(viii) $(S S]=S=S S$, if $S$ has a left identity.

Lemma 7. Let $R$ be a right ideal and $L$ be a left ideal of an ordered $\mathcal{A}^{*} \mathcal{G}^{* *}$-groupoid $S$. Then $(R L]$ is a left ideal of $S$.

ProofLet $R$ be a right ideal and $L$ be a left ideal of $S$. Then by using Lemma 6 , we get $S(R L]=(S S](R L] \subseteq(S S \cdot R L]=$ $(S R \cdot S L] \subseteq(S R \cdot(S L]]=(S R \cdot L]=((S S] R \cdot L] \subseteq$ $((S S) R \cdot L]=((R S) S \cdot L] \subseteq((R S] S \cdot L] \subseteq(R L]$, which shows that $(R L]$ is a left ideal of $S$.

The converse inclusion of above Lemma is not true in general. Let us consider an ordered $\mathcal{A}^{*} \mathcal{G}^{* *}$-groupoid $(S, \cdot, \leq)$ defined as follows:

\begin{tabular}{c|ccccc}
$\cdot$ & $a$ & $b$ & $c$ & $d$ & $e$ \\
\hline$a$ & $b$ & $b$ & $d$ & $d$ & $e$ \\
$b$ & $b$ & $b$ & $b$ & $b$ & $e$ \\
$c$ & $a$ & $b$ & $c$ & $d$ & $e$ \\
$d$ & $a$ & $b$ & $a$ & $b$ & $e$ \\
$e$ & $a$ & $e$ & $e$ & $e$ & $e$
\end{tabular}

$\leq=\{(a, a),(a, b),(c, c),(a, c),(d, d),(a, e),(e, e),(b, b)\}$. 
Setting $R=\{a, b\}$ and $L=\{d, e\}$. Then it is easy to verify that $(R L]$ is a left ideal of $S$ but $R$ (resp. $L$ ) is not a right (resp. left) ideal of $S$.

- Recall that an ordered $\mathcal{A G}^{* *}$-groupoid is an ordered $\mathcal{A G}$ groupoid in which $a \cdot b c=b \cdot a c, \forall a, b, c \in S$. Note that an ordered $\mathcal{A G}^{* *}$-groupoid satisfies the paramedial law as well.

Now let us introduce the concept of an ordered $\mathcal{A}^{*} \mathcal{G}^{* *}$ groupoid as follows:

- An ordered $\mathcal{A G}^{* *}$-groupoid $S$ is called an ordered $\mathcal{A}^{*} \mathcal{G}^{* *}$ groupoid if $S=S^{2}$.

Example 4. Let $S=\{x, y, z\}$ be an ordered $\mathcal{A G}$ groupoiddefine in the following multiplication table and ordered below.

$$
\begin{aligned}
& \begin{array}{l|lll}
\cdot & x & y & z \\
\hline x & z & y & z \\
y & x & z & z \\
z & z & z & z
\end{array} \\
& \leq:=\{(x, x),(y, y),(z, z),(z, x),(z, y)\}
\end{aligned}
$$

It is easy to verify that $S$ is an ordered $\mathcal{A}^{*} \mathcal{G}^{* *}$-groupoid.

Note that every ordered $\mathcal{A G}$-groupoid (ordered $\mathcal{A G}^{* *}$ groupoid) with left identity is an ordered $\mathcal{A}^{*} \mathcal{G}^{* *}$-groupoid but the converse is not true in general. An ordered $\mathcal{A G}$-groupoid (ordered $\mathcal{A G}^{* *}$-groupoid) in above example is an ordered $\mathcal{A}^{*} \mathcal{G}^{* *}$-groupoid but it does not contains a left identity. Hence we can say that an ordered $\mathcal{A}^{*} \mathcal{G}^{* *}$-groupoid is the generalization of an ordered $\mathcal{A G}$-groupoid (ordered $\mathcal{A G}^{* *}$-groupoid) with left identity.

Now let us define the generated left and right ideals of an ordered $\mathcal{A}^{*} \mathcal{G}^{* *}$-groupoid as follows:

Lemma 8. Let $S$ be an ordered $\mathcal{A}^{*} \mathcal{G}^{* *}$-groupoid and $a \in S$. Then $\langle R\rangle_{a^{2}}=\left(S a^{2} \cup a^{2}\right]\left(\right.$ resp. $\left.\langle L\rangle_{a}=(S a \cup a]\right)$ is the right (resp. left) ideal of $S$ containing $a^{2}$ (resp. $\left.a\right)$.

Proof Let $a \in S$, then by using Lemma 6 , we get $\left(S a^{2} \cup\right.$ $\left.a^{2}\right] S=\left(S a^{2} \cup a^{2}\right](S]=\left(\left(S a^{2} \cup a^{2}\right) S\right]=\left(S a^{2} \cdot S \cup a^{2} S\right]=$ $\left(S S \cdot a^{2} S \cup S S \cdot a a\right]=\left(S \cdot a^{2} S \cup S a^{2}\right)=\left(a^{2} \cdot S S \cup S a^{2}\right]=$ $\left(S a^{2}\right] \subseteq\left(S a^{2} \cup a^{2}\right]$, which is what we set out to prove. Similarly we can prove that $S(S a \cup a] \subseteq(S a \cup a]$.

From now onward, $E$ will denote the set of all idempotents of an ordered $\mathcal{A}^{*} \mathcal{G}^{* *}$-groupoid, that is, $E=\left\{x \in S: x=x^{2}\right\}$.

Lemma 9. Let $S$ be an ordered $\mathcal{A}^{*} \mathcal{G}^{* *}$-groupoid and $\emptyset \neq$ $E \subseteq S$. Then the following assertions hold:

(i) $E$ forms a semilattice ;

(ii) $E$ is a singleton set, if $a=a x \cdot a, \forall a, x \in S$.

Proof It is simple.

- An element $a$ of an ordered $\mathcal{A G}$-groupoid $S$ is called a $(2,2)$-regular element of $S$, if there exists some $x$ in $S$ such that $a \leq a^{2} x \cdot a^{2}$, and $S$ is called $(2,2)$-regular ordered
$\mathcal{A G}$-groupoid if all elements of $S$ are $(2,2)$-regular.

Let us characterize a $(2,2)$-regular element of an ordered $\mathcal{A}^{*} \mathcal{G}^{* *}$-groupoid as follows:

Theorem 4. Let $S$ be an ordered $\mathcal{A}^{*} \mathcal{G}^{* *}$-groupoid. An element $a$ of $S$ is $(2,2)$-regular if and only if for all $a \in S$, $a \leq a y \cdot a z$ for some $y, z \in S(a \leq a t \cdot a, a t=t a$ for some $t \in S)$.

Proof Necessity. Let $a \in S$ is (2,2)-regular, then $a \leq a^{2} x$. $a^{2}=a^{2} \cdot x a^{2}=a a \cdot a(x a)=a a \cdot a y$, where $x a=z \in S$. Thus $a \leq a y \cdot a z$ for some $y, z \in S$. Also $a \leq a^{2} x \cdot a^{2}=$ $a a \cdot x a^{2}=\left(x a^{2} \cdot a\right) a=t a \cdot a$, where $x a^{2} \cdot a=t \in S$. Thus $t a \leq t(t a \cdot a)=t a \cdot t a=(t a \cdot a) t \leq a t$, and $a \leq t a \cdot a \leq a t \cdot a$.

Sufficiency. Let $a \in S$ such that $a \leq a x \cdot a y$ for some $x, y \in S$. Then $a \leq a x \cdot a y \leq(a x \cdot a y) x \cdot(a x \cdot a y) y=$ $\left(a^{2} \cdot x y\right) x \cdot\left(a^{2} \cdot x y\right) y=(x \cdot x y) a^{2} \cdot\left(a^{2} \cdot x y\right) y=$ $a^{2}(x y \cdot x) \cdot\left(a^{2} \cdot x y\right) y=\left(\left(a^{2} \cdot x y\right) y \cdot(x y \cdot x)\right) a^{2}=$ $\left((y \cdot x y) a^{2} \cdot(x y \cdot x)\right) a^{2}=\left(a^{2}\left(y^{2} x\right) \cdot(x y \cdot x)\right) a^{2}=((x \cdot x y)$. $\left.\left(y^{2} x\right) a^{2}\right) a^{2}=\left((x \cdot x y) \cdot a^{2}\left(x y^{2}\right)\right) a^{2}=\left(a^{2} \cdot(x \cdot x y)\left(x y^{2}\right)\right) a^{2}$, where $(x \cdot x y)\left(x y^{2}\right)=u \in S$. The remaining part is simple. Hence $S$ is $(2,2)$-regular.

Now let us characterize a $(2,2)$-regular class of an ordered $\mathcal{A}^{*} \mathcal{G}^{* *}$-groupoid in terms of its semilattice $E$ as follows:

Theorem 5. A $(2,2)$-regular ordered $\mathcal{A} \mathcal{G}$-groupoid is an ordered $\mathcal{A}^{*} \mathcal{G}^{* *}$-groupoid if and only if $E$ is a semilattice.

Proof Necessity. It can be followed from Lemma $9(i)$.

Sufficiency. Let $a, b, c \in S$, then there exist $a^{\prime}, b^{\prime}, c^{\prime} \in S$ such that $a \cdot b c \leq\left(a a^{\prime} \cdot b b^{\prime} \cdot c c^{\prime}\right)(a \cdot b c)$, as clearly $a a^{\prime}, b b^{\prime}, c c^{\prime} \in$ $E$. Therefore $a \cdot b c \leq\left(a a^{\prime} \cdot a\right)(b c)=(b c \cdot a)\left(a a^{\prime}\right)=(a c$. $b)\left(a a^{\prime}\right)=\left(a a^{\prime} \cdot b\right)(a c) \leq\left(a a^{\prime} \cdot b\right) \cdot\left(a a^{\prime} \cdot a\right)\left(c c^{\prime} \cdot c\right)=\left(a a^{\prime}\right.$. b) $\cdot\left(a a^{\prime} \cdot c c^{\prime}\right)(a c)=\left(a a^{\prime} \cdot a a^{\prime} \cdot c c^{\prime}\right) \cdot(b \cdot a c) \leq\left(a a^{\prime} \cdot a a^{\prime} \cdot c c^{\prime}\right)$. $\left(b b^{\prime} \cdot a a^{\prime} \cdot c c^{\prime}\right)(b \cdot a c)=\left(a a^{\prime} \cdot b b^{\prime} \cdot a a^{\prime} \cdot c c^{\prime}\right) \cdot\left(a a^{\prime} \cdot c c^{\prime}\right)(b \cdot a c)=$ $\left(b b^{\prime} \cdot b b^{\prime} \cdot a a^{\prime} \cdot c c^{\prime}\right) \cdot\left(a a^{\prime} \cdot c c^{\prime}\right)(b \cdot a c)=\left(b b^{\prime} \cdot a a^{\prime} \cdot c c^{\prime}\right) \cdot\left(b b^{\prime}\right.$. $\left.a a^{\prime} \cdot c c^{\prime}\right)(b \cdot a c)=\left(b b^{\prime} \cdot a a^{\prime} \cdot c c^{\prime}\right)(b \cdot a c) \leq b \cdot a c$. Also it is easy to show that $S=S^{2}$ if $S$ is $(2,2)$-regular. Hence $S$ is an ordered $\mathcal{A}^{*} \mathcal{G}^{* *}$-groupoid.

\subsection{Characterization Problems}

In this section, we generalize the results of an ordered semigroup and get some interesting characterizations which we usually do not find in other algebraic structures.

Let $S$ be an ordered $\mathcal{A}^{*} \mathcal{G}^{* *}$-groupoid. From now onward, $R$ (resp. L) will denote any right (resp. left) ideal of $S ;\langle R\rangle_{a^{2}}$ will denote a right ideal $\left(S a^{2} \cup a^{2}\right]$ of $S$ containing $a^{2}$ and $\langle L\rangle_{a}$ will denote a left ideal $(S a \cup a]$ of $S$ containing $a ; f$ (resp. $g$ ) will denote any $\left(\in_{\gamma}, \in_{\gamma} \vee q_{\delta}\right)$-fuzzy right (resp. left) ideal of $S$ unless otherwise specified.

We characterize a $(2,2)$-regular class of an ordered $\mathcal{A}^{*} \mathcal{G}^{* *}$ groupoid in terms of its generated right/left ideals as follows:

Theorem 6. Let $S$ be an ordered $\mathcal{A}^{*} \mathcal{G}^{* *}$-groupoid. Then $S$ is $(2,2)$-regular if and only if $\langle R\rangle_{a^{2}} \cap\langle L\rangle_{a}=\left(\langle R\rangle_{a^{2}}^{2}\langle L\rangle_{a}^{2}\right]$ and $\langle R\rangle_{a^{2}}$ is semiprime. 
Proof Necessity: Let $S$ be $(2,2)$-regular. It is easy to see that $\left(\langle R\rangle_{a^{2}}^{2}\langle L\rangle_{a}^{2}\right] \subseteq\langle R\rangle_{a^{2}} \cap\langle L\rangle_{a}$. Let $a \in\langle R\rangle_{a^{2}} \cap\langle L\rangle_{a}$. Then there exist some $x, y \in S$ such that $a \leq a x \cdot a y \leq$ $(a x \cdot a y) x \cdot(a x \cdot a y) y=(x \cdot a y)(a x) \cdot(y \cdot a y)(a x)=(a$ $x y)(a x) \cdot\left(a y^{2}\right)(a x)=(a \cdot x y)(a x) \cdot(x a)\left(y^{2} a\right) \in\left(\langle R\rangle_{a^{2}} S\right.$. $\left.\langle R\rangle_{a^{2}} S\right)\left(S\langle L\rangle_{a} \cdot S\langle L\rangle_{a}\right) \subseteq\langle R\rangle_{a^{2}}^{2}\langle L\rangle_{a}^{2}$, which shows that $\langle R\rangle_{a^{2}} \cap\langle L\rangle_{a}=\left(\langle R\rangle_{a^{2}}^{2}\langle L\rangle_{a}^{2}\right]$. It is easy to see that $\langle R\rangle_{a^{2}}$ is semiprime.

Sufficiency: Since $\left(S a^{2} \cup a^{2}\right]$ and $(S a \cup a]$ are the right and left ideals of $S$ containing $a^{2}$ and $a$ respectively. Thus by using given assumption and Lemma 6 , we get $a \in\left(S a^{2} \cup a^{2}\right] \cap(S a \cup a]=\left(\left(S a^{2} \cup a^{2}\right]^{2}(S a \cup a]^{2}\right]=\left(\left(S a^{2} \cup\right.\right.$ $\left.\left.a^{2}\right)\left(S a^{2} \cup a\right) \cdot(S a \cup a)(S a \cup a)\right] \subseteq\left(S\left(S a^{2} \cup a\right) \cdot S(S a \cup a)\right]=$ $\left(\left(S \cdot S a^{2} \cup S a\right)(S \cdot S a \cup S a)\right]=\left(\left(a^{2} S \cdot S \cup S a\right)(a S \cdot S \cup S a)\right]=$ $\left(\left(a^{2} S \cdot S \cup S a\right)(a S \cdot S \cup S a)\right]=\left(\left(S a^{2} \cup S a\right)(S a \cup S a)\right]=$ $\left(\left(a^{2} S \cup S a\right)(S a \cup S a)\right]=((S a \cdot a \cup S a)(S a \cup S a)] \subseteq$ $((S a \cup S a)(S a \cup S a)]=(S a \cdot S a]=(a S \cdot a S]$. This implies that $S$ is $(2,2)$-regular.

Lemma 10. Let $I$ be a non-empty subset of a $(2,2)$-regular ordered $\mathcal{A}^{*} \mathcal{G}^{* *}$-groupoid $S$. Then $I$ is a left ideal of $S$ if and only if it is a right ideal of $S$.

proof. It is simple.

Corollary 2. Let $S$ be an ordered $\mathcal{A}^{*} \mathcal{G}^{* *}$-groupoid. Then $S$ is $(2,2)$-regular if and only if $\langle R\rangle_{a^{2}} \cap\langle L\rangle_{a}=\left(\langle L\rangle_{a}^{2}\langle R\rangle_{a^{2}}^{2}\right]$ and $\langle R\rangle_{a^{2}}$ is semiprime.
Lemma 11. Let $f$ be any $\left(\epsilon_{\gamma}, \in_{\gamma} \vee q_{\delta}\right)$-fuzzy right (left) ideal of a $(2,2)$-regular ordered $\mathcal{A}^{*} \mathcal{G}^{* *}$-groupoid $S$. Then the following assertions hold:

(i) $f={ }_{(\gamma, \delta)} f \circ S\left(f={ }_{(\gamma, \delta)} S \circ f\right)$;

(ii) $f$ is $\left(\in_{\gamma}, \in_{\gamma} \vee q_{\delta}\right)$-fuzzy semiprime.

Proof. It is simple.

Theorem 7. Let $f, g$ be any $\left(\epsilon_{\gamma}, \in_{\gamma} \vee q_{\delta}\right)$-fuzzy left ideals of an ordered $\mathcal{A}^{*} \mathcal{G}^{* *}$-groupoid $S$. Then the following conditions are equivalent:

(i) $S$ is $(2,2)$-regular;

(ii) $\left(\langle R\rangle_{a^{2}}\langle L\rangle_{a}\right] \cap\langle L\rangle_{a}=\left(\left(\langle R\rangle_{a^{2}} \cdot\langle R\rangle_{a^{2}}\langle L\rangle_{a}\right)\langle L\rangle_{a} \cdot\langle L\rangle_{a}\right]$ and $\langle R\rangle_{a^{2}}\left(\right.$ resp. $\left.\langle L\rangle_{a}\right)$ is semiprime;

(iii) $(R L] \cap L=((R \cdot R L) L \cdot L]$ and $R$ (resp. $L)$ is semiprime;

(iv) $f \cap g={ }_{(\gamma, \delta)}(f \circ g) \circ f$ and $f($ resp.g $)$ is $\left(\in_{\gamma}, \in_{\gamma} \vee q_{\delta}\right)$ fuzzy semiprime;

(v) $S$ is $(2,2)$-regular and $|E|=1,(a=a x \cdot a, \forall a, x \in E)$;

(vi) $S$ is $(2,2)$-regular and $\emptyset \neq E \subseteq S$ is semilattice.

Proof. $(i) \Longrightarrow(v i)$ : It can be followed from Lemma $9(i)$.

$(v i) \Longrightarrow(v)$ : It can be followed from Lemma $9(i i)$.

$(v) \Longrightarrow(i v)$ : Let $f$ and $g$ be any $\left(\in_{\gamma}, \in_{\gamma} \vee q_{\delta}\right)$-fuzzy left ideals of a $(2,2)$-regular $S$. Now for $a \in S$, there exist some $x, y \in S$ such that $a \leq a x \cdot a y=y a \cdot x a \leq y(a x \cdot a y) \cdot x a=$ $(a x)(y \cdot a y) \cdot x a=(a y \cdot y)(x a) \cdot x a=\left(y^{2} a \cdot x a\right)(x a)$. Thus $\left(y^{2} a \cdot x a, x a\right) \in A_{a}$. Therefore

$$
\begin{aligned}
\max \{((f \circ g) \circ f)(a), \gamma\} & =\max \left[\bigvee_{\left(y^{2} a \cdot x a, x a\right) \in A_{a}}\left\{(f \circ g)\left(y^{2} a \cdot x a\right) \wedge f(x a)\right\}, \gamma\right] \\
& \geq \max \left[\min \left\{(f \circ g)\left(y^{2} a \cdot x a\right), f(x a)\right\}, \gamma\right] \\
& =\min \left[\max \left\{(f \circ g)\left(y^{2} a \cdot x a\right), \gamma\right\}, \max \{f(x a), \gamma\}\right] \\
& =\min \left[\max \left\{\underset{\left(y^{2} a \cdot x a, x a\right) \in A_{a}}{\bigvee}\left\{f\left(y^{2} a \cdot x a\right) \wedge g(x a), \gamma\right\}\right\}, \max \{f(x a), \gamma\}\right] \\
& \geq \min \left[\max \left\{f\left(y^{2} a \cdot x a\right) \wedge g(x a), \gamma\right\}, \max \{f(x a), \gamma\}\right] \\
& =\min \left[\max \left\{\min \left\{f\left(y^{2} a \cdot x a\right), g(x a)\right\}, \gamma\right\}, \max \{f(x a), \gamma\}\right] \\
& =\min \left[\max \left\{\max \left\{f\left(y^{2} a \cdot x a\right), \gamma\right\}, \max \{g(x a), \gamma\}\right\}, \max \{f(x a), \gamma\}\right] \\
& \geq \min [\min \{f(a) \wedge g(a), \delta\}, \min \{f(x a), \delta\}] \\
& =\min \{(f \cap g)(a), \delta\},
\end{aligned}
$$

which shows that $(f \circ g) \circ f \supseteq(\gamma, \delta) f \cap g$. By using Lemmas 3 and 11, it is easy to show that $(f \circ g) \circ f \subseteq_{(\gamma, \delta)} f \cap g$. Thus $f \cap g={ }_{(\gamma, \delta)}(f \circ g) \circ f$. Also by using Lemma 11, $f$ (resp. $\left.g\right)$ is $\left(\in_{\gamma}, \in_{\gamma} \vee q_{\delta}\right)$-fuzzy semiprime.

$(i v) \Longrightarrow($ iii $)$ : Let $R$ and $L$ be any right and left ideals of $S$ respectively. Then by using Lemmas 4 and $7, \mathcal{X}_{(R L]}$ and $\mathcal{X}_{L}$ are the $\left(\epsilon_{\gamma}, \in_{\gamma} \vee q_{\delta}\right)$-fuzzy left ideals of $S$. Now by using Lemma 2, we get $\mathcal{X}_{(R L] \cap L}=\mathcal{X}_{(R L]} \cap \mathcal{X}_{L}=\left(\mathcal{X}_{(R L]} \circ \mathcal{X}_{L}\right) \circ \mathcal{X}_{(R L]}=$
$\mathcal{X}_{((R L] L \cdot(R L]]}$, which give us $(R L] \cap L=((R L] L \cdot(R L])$. Now by using Lemma 6 , we get $((R L] L \cdot(R L]]=((R L) L \cdot R L]=$ $\left(L^{2} R \cdot R L\right]=\left(L R \cdot R L^{2}\right]=\left(R\left(L R \cdot L^{2}\right)\right]=\left(R\left(L^{2} \cdot R L\right)\right]=$ $\left(R\left(R \cdot L^{2} L\right)\right]=\left(R \cdot R L^{3}\right]=\left(R\left(R \cdot L^{2} L\right)\right]=\left(R\left(L^{2} \cdot R L\right)\right]=$ $((R \cdot R L) L \cdot L]$. Also by using Lemma $5, L$ is semiprime. It is easy to show that every right ideal of an ordered $\mathcal{A}^{*} \mathcal{G}^{* *}$ groupoid $S$ is a left ideal of $S$. Thus $R$ is also semiprime.

$($ iii $) \Longrightarrow(i i)$ : It is simple.

$(i i) \Longrightarrow(i)$ : Since $\left(S a^{2} \cup a^{2}\right]$ and $(S a \cup a]$ are the right 
and left ideals of $S$ containing $a^{2}$ and $a$ respectively. Thus by using given assumption and Lemma 6, we get

$$
\begin{aligned}
a & \in\left(\left(S a^{2} \cup a^{2}\right](S a \cup a]\right] \cap(S a \cup a] \\
& \left.=\left(\left(S a^{2} \cup a^{2}\right] \cdot\left(S a^{2} \cup a^{2}\right](S a \cup a]\right)(S a \cup a] \cdot(S a \cup a]\right] \\
& \subseteq\left(\left(\left(S a^{2} \cup a^{2}\right) \cdot\left(S a^{2} \cup a^{2}\right)(S a \cup a)\right)(S a \cup a) \cdot(S a \cup a)\right] \\
& \subseteq\left(\left(\left(S a^{2} \cup a^{2}\right) \cdot S\right) S \cdot(S a \cup a)\right]=\left((S S)\left(S a^{2} \cup a^{2}\right) \cdot(S a \cup a)\right] \\
& =\left(S\left(S a^{2} \cup a^{2}\right) \cdot(S a \cup a)\right]=\left(\left(S \cdot S a^{2} \cup S a^{2}\right)(S a \cup a)\right] \\
& =\left(\left(S a^{2} \cup S a^{2}\right)(S a \cup a)\right]=\left(\left(S a^{2}\right)(S a \cup a)\right]=\left(S a^{2} \cdot S a \cup S a^{2} \cdot a\right] \\
& =\left(a^{2} S \cdot S a \cup a^{2} S \cdot a\right]=((S a \cdot a)(S a) \cup(S a \cdot a) a] \subseteq(S a \cdot S a \cup(S a \cdot a) a] \\
& =(S a \cdot S a \cup a a \cdot S a] \subseteq(S a \cdot S a \cup S a \cdot S a]=(S a \cdot S a]=(a S \cdot a S] .
\end{aligned}
$$

Hence $S$ is $(2,2)$-regular.

Theorem 8. Let $S$ be an ordered $\mathcal{A}^{*} \mathcal{G}^{* *}$-groupoid. Then the following conditions are equivalent:

(i) $S$ is $(2,2)$-regular;

(ii) $\langle R\rangle_{a^{2}} \cap\langle L\rangle_{a}=\left(\langle R\rangle_{a^{2}}\left(\langle L\rangle_{a}\langle R\rangle_{a^{2}} \cdot\langle R\rangle_{a^{2}}\right)\right]$ and $\langle R\rangle_{a^{2}}$ is semiprime;

(iii) $R \cap L=(R(L R \cdot R)]$ and $R$ is semiprime;

(iv) $f \cap g={ }_{(\gamma, \delta)} f^{3} \circ g$ and $f$ is $\left(\in_{\gamma}, \in_{\gamma} \vee q_{\delta}\right)$-fuzzy semiprime;

(v) $S$ is (2,2)-regular and $|E|=1,(a=a x \cdot a, \forall a, x \in E)$;

(vi) $S$ is $(2,2)$-regular and $\emptyset \neq E \subseteq S$ is semilattice.

Proof. $(i) \Longrightarrow(v i)$ : It can be followed from Lemma $9(i)$.

$(v i) \Longrightarrow(v)$ : It can be followed from Lemma $9(i i)$.

$(v) \Longrightarrow(i v)$ : Let $f$ and $g$ be any $\left(\in_{\gamma}, \in_{\gamma} \vee q_{\delta}\right)$-fuzzy right ideal and $\left(\epsilon_{\gamma}, \in_{\gamma} \vee q_{\delta}\right)$-fuzzy left ideal of a $(2,2)$-regular $S$ respectively. From Lemma 3, it is easy to show that $f^{3} \circ g \subseteq(\gamma, \delta)$ $f \cap g$. Now for $a \in S$, there exist some $x, y \in S$ such that $a \leq a x \cdot a y \leq(a x \cdot a y) x \cdot(a x \cdot a y) y=y(a x \cdot a y) \cdot x(a x \cdot a y)=$ $(a x)(y \cdot a y) \cdot(a x)(x \cdot a y)=(a x)\left(a y^{2}\right) \cdot(a x)(a \cdot x y)=$ $\left(y^{2} a\right)(x a) \cdot(a x)(a \cdot x y)=((a x)(a \cdot x y))(x a) \cdot y^{2} a=$ $((a x)(a \cdot x y))(e x \cdot a) \cdot y^{2} a=((a x)(a \cdot x y))(a x \cdot e) \cdot y^{2} a=$ $b c \cdot y^{2} a=d \cdot y^{2} a$, where $d=b c=((a x)(a \cdot x y))(a x \cdot e)$. Thus

$$
\begin{aligned}
& \max \{((f \circ f) \circ f)(d), \gamma\} \\
& =\max \left[\bigvee_{d \leq b c}\{(f \circ f)(b) \wedge f(c)\}, \gamma\right] \\
& \geq \max [\min \{(f \circ f)(b), f(c)\}, \gamma] \\
& =\min [\max \{(f \circ f)(b), \gamma\}, \max \{f(c), \gamma\}] \\
& =\min \left[\max \left\{\begin{array}{c}
\bigvee \\
b \leq(a x)(a \cdot x y) \\
, \max \{f(c), \gamma\}
\end{array}\right]\right.
\end{aligned}
$$

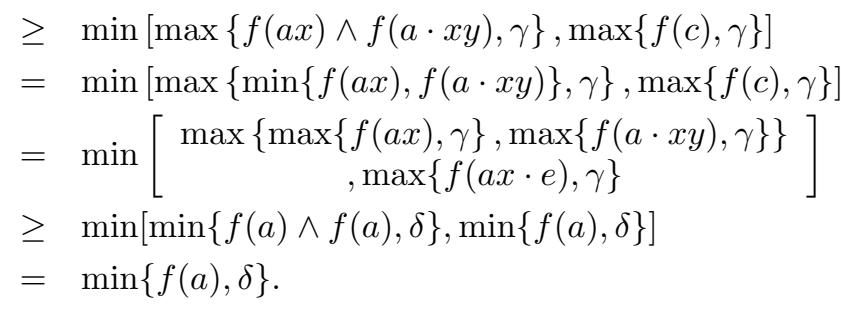

Therefore

$$
\begin{aligned}
& \max \left\{\left(f^{3} \circ g\right)(a), \gamma\right\} \\
& =\max \left[\begin{array}{c}
\bigvee_{a \leq d \cdot y^{2} a}\{((f \circ f) \circ f)(((a x)(a \cdot x y))(a x \cdot e)) \\
\left.\wedge g\left(y^{2} a\right)\right\}, \gamma
\end{array}\right] \\
& \geq \min \{(f \cap g)(a), \delta\},
\end{aligned}
$$

which shows that $f \cap g \subseteq{ }_{(\gamma, \delta)} f^{3} \circ g$. Thus $f \cap g=_{(\gamma, \delta)} f^{3} \circ g$. Also by using Lemma 11, $f$ is $\left(\in_{\gamma}, \in_{\gamma} \vee q_{\delta}\right)$-fuzzy semiprime.

$(i v) \Longrightarrow($ iii $)$ : Let $R$ and $L$ be any right and left ideals of $S$ respectively. Then by using Lemma $4, \mathcal{X}_{R}$ and $\mathcal{X}_{L}$ are the $\left(\epsilon_{\gamma}, \epsilon_{\gamma} \vee q_{\delta}\right)$-fuzzy right ideal and $\left(\epsilon_{\gamma}, \epsilon_{\gamma} \vee q_{\delta}\right)$-fuzzy left ideal of $S$ respectively. Now by using Lemma 2, we get $\mathcal{X}_{R \cap L}=\mathcal{X}_{R} \cap \mathcal{X}_{L}=\left(\left(\mathcal{X}_{R} \circ \mathcal{X}_{R}\right) \circ \mathcal{X}_{R}\right) \circ \mathcal{X}_{L}=\mathcal{X}_{\left(R^{3}\right]} \circ \mathcal{X}_{L}=$ $\mathcal{X}_{\left(\left(R^{3}\right] L\right]}$, which implies that $R \cap L=\left(\left(R^{3}\right] L\right]$. Now by using Lemma ref6, we get $R \cap L=\left(\left(R^{3}\right] L\right]=\left(R^{3} L\right]=\left(R^{2} R \cdot L\right]=$ $\left(L R \cdot R^{2}\right]=\left(R^{2} \cdot R L\right]=\left(R \cdot R^{2} L\right]=(R(L R \cdot R)]$. Also by using Lemma $5, R$ is semiprime.

$$
(\text { iii }) \Longrightarrow(i i) \text { : It is simple. }
$$

$(i i) \Longrightarrow(i)$ : Since $\left(S a^{2} \cup a^{2}\right]$ and $(S a \cup a]$ are the right and left ideals of $S$ containing $a^{2}$ and $a$ respectively. Thus by using given assumption and Lemma 6 , we get 


$$
\begin{aligned}
a & \in\left(S a^{2} \cup a^{2}\right] \cap(S a \cup a] \\
& =\left(\left(S a^{2} \cup a^{2}\right]\left((S a \cup a]\left(S a^{2} \cup a^{2}\right] \cdot\left(S a^{2} \cup a^{2}\right]\right)\right] \\
& \subseteq\left(\left(S a^{2} \cup a^{2}\right)\left((S a \cup a)\left(S a^{2} \cup a^{2}\right) \cdot\left(S a^{2} \cup a^{2}\right)\right)\right] \\
& =\left((S a \cup a)\left(S a^{2} \cup a^{2}\right) \cdot\left(S a^{2} \cup a^{2}\right)\left(S a^{2} \cup a^{2}\right)\right] \\
& \subseteq\left(S \cdot S\left(S a^{2} \cup a^{2}\right)\right] \\
& \left.=\left(S\left(S \cdot S a^{2}\right) \cup S \cdot S a^{2}\right)\right] \\
& \left.\left.=\left(\left(S a^{2} \cdot S\right) S \cup S \cdot S a^{2}\right)\right]=\left(S \cdot S a^{2} \cup S \cdot S a^{2}\right)\right] \\
& =\left(S \cdot S a^{2}\right]=\left(a^{2} S \cdot S\right]=(S S \cdot a a]=(S a \cdot S a] \\
& =(a S \cdot a S] .
\end{aligned}
$$

Hence $S$ is $(2,2)$-regular.

We characterize a $(2,2)$-regular class of an ordered $\mathcal{A}^{*} \mathcal{G}^{* *}$ groupoid in terms of its $\left(\in_{\gamma}, \in_{\gamma} \vee q_{\delta}\right)$-fuzzy right ideal $f$ and $\left(\in_{\gamma}, \in_{\gamma} \vee q_{\delta}\right)$-fuzzy left ideal $g$ as follows:

Corollary 3. Let $S$ be an ordered $\mathcal{A}^{*} \mathcal{G}^{* *}$-groupoid. Then $S$ is $(2,2)$-regular if and only if $f^{3} \circ g={ }_{(\gamma, \delta)} f \cap g={ }_{(\gamma, \delta)} g^{3} \circ f$ and $f$ is $\left(\in_{\gamma}, \in_{\gamma} \vee q_{\delta}\right)$-fuzzy semiprime.

Proof. Necessity. From Theorem 8 , it follows that $f^{3}$ 。 $g={ }_{(\gamma, \delta)} f \cap g$ and $f$ is $\left(\in_{\gamma}, \in_{\gamma} \vee q_{\delta}\right)$-fuzzy semiprime. Similarly we can show that $g^{3} \circ f={ }_{(\gamma, \delta)} f \cap g$.

Sufficiency. It can be followed from Theorem 8 as well.

Theorem 9.Let $S$ be an ordered $\mathcal{A}^{*} \mathcal{G}^{* *}$-groupoid. Then the following conditions are equivalent:

(i) $S$ is (2,2)-regular;

(ii) $f \cap g={ }_{(\gamma, \delta)}\left(f^{3} \circ g\right) \cap\left(g^{3} \circ f\right)$ and $f$ is $\left(\in_{\gamma}, \in_{\gamma} \vee q_{\delta}\right)$ fuzzy semiprime (for any $\left(\in_{\gamma}, \in_{\gamma} \vee q_{\delta}\right)$-fuzzy right ideal $f$ and $\left(\in_{\gamma}, \in_{\gamma} \vee q_{\delta}\right)$-fuzzy left ideal $g$ of $\left.S\right)$;

(iii) $f \cap g={ }_{(\gamma, \delta)}\left(f^{3} \circ g\right) \cap\left(g^{3} \circ f\right)$ and $f$ is $\left(\in_{\gamma}, \in_{\gamma} \vee q_{\delta}\right)$ fuzzy semiprime (for any $\left(\in_{\gamma}, \in_{\gamma} \vee q_{\delta}\right)$-fuzzy right ideal $f$ and $\left(\in_{\gamma}, \in_{\gamma} \vee q_{\delta}\right)$-fuzzy bi-ideal $g$ of $\left.S\right)$;

(iv) $f \cap g={ }_{(\gamma, \delta)}\left(f^{3} \circ g\right) \cap\left(g^{3} \circ f\right)$ and $f$ is $\left(\in_{\gamma}, \in_{\gamma} \vee q_{\delta}\right)$ fuzzy semiprime (for any $\left(\in_{\gamma}, \in_{\gamma} \vee q_{\delta}\right)$-fuzzy right ideal $f$ and $\left(\in_{\gamma}, \in_{\gamma} \vee q_{\delta}\right)$-fuzzy generalized bi-ideal $g$ of $\left.S\right)$;

$(v) f \cap g={ }_{(\gamma, \delta)}\left(f^{3} \circ g\right) \cap\left(g^{3} \circ f\right)$ and $f, g$ are $\left(\in_{\gamma}, \in_{\gamma} \vee q_{\delta}\right)$ fuzzy semiprime (for $\left(\in_{\gamma}, \in_{\gamma} \vee q_{\delta}\right)$-fuzzy bi-ideals $f, g$ of $S$ );

(vi) $f \cap g={ }_{(\gamma, \delta)}\left(f^{3} \circ g\right) \cap\left(g^{3} \circ f\right)$ and $f, g$ are $\left(\in_{\gamma}, \in_{\gamma} \vee q_{\delta}\right)$ fuzzy semiprime (for $\left(\in_{\gamma}, \in_{\gamma} \vee q_{\delta}\right)$-fuzzy generalized bi-ideals $f, g$ of $S)$; $E)$;

(vii) $S$ is $(2,2)$-regular and $|E|=1,(a=a x \cdot a, \forall a, x \in$

(viii) $S$ is $(2,2)$-regular and $\emptyset \neq E \subseteq S$ is semilattice.

Proof. $(i) \Longrightarrow($ viii $)$ : It can be followed from Lemma 9 (i).

(viii) $\Longrightarrow($ vii $)$ : It can be followed from Lemma $9(i i)$.

(vii) $\Longrightarrow(v i)$ : Let $f$ and $g$ be the $\left(\in_{\gamma}, \in_{\gamma} \vee q_{\delta}\right)$-fuzzy generalized bi-ideals of $S$. Now for $a \in S$, there exist $x, y \in S$ such that

$$
\begin{aligned}
& a \leq a x \cdot a y \leq(a x \cdot a y) x \cdot(a x \cdot a y) y \\
& =(a x \cdot a y)(a x \cdot a y) \cdot x y \\
& =(a x \cdot a y)(a a \cdot x y) \cdot x y=(a a)((a x \cdot a y)(x y)) \cdot x y \\
& =((x y)(a x \cdot a y))(a a) \cdot x y=a(((x y)(a x \cdot a y)) a) \cdot x y \\
& =(x y)(((x y)(a x \cdot a y)) a) \cdot a=(x y)(a(a x \cdot a y) \cdot x y) \cdot a \\
& =(x y)(a x \cdot(a x \cdot a y) y) \cdot a=(x y)(a x \cdot(y \cdot a y)(a x)) \cdot a \\
& =(a x)(x y \cdot(y \cdot a y)(a x)) \cdot a=\left(\left(x y \cdot\left(a y^{2}\right)(a x)\right) x\right) a \cdot a \\
& =\left(\left(x \cdot\left(a y^{2}\right)(a x)\right) \cdot x y\right) a \cdot a=\left(\left(x \cdot(a a)\left(y^{2} x\right)\right) \cdot x y\right) a \cdot a \\
& =\left(\left(a a \cdot x\left(y^{2} x\right)\right) \cdot x y\right) a \cdot a=\left(\left(a a \cdot y^{2} x^{2}\right) \cdot x y\right) a \cdot a \\
& =\left(\left(x y \cdot y^{2} x^{2}\right) \cdot a a\right) a \cdot a=\left(\left(x y \cdot x^{2} y^{2}\right) \cdot a a\right) a \cdot a \\
& =\left(\left(y^{2} x^{2} \cdot y x\right) \cdot a a\right) a \cdot a=\left(y^{3} x^{3} \cdot a a\right) a \cdot a \\
& =\left(a a \cdot x^{3} y^{3}\right) a \cdot a \\
& =\left(\left(x^{3} y^{3} \cdot a\right) a\right) a \cdot a=\left(\left(x^{3} y^{3} \cdot(a x \cdot a y)\right) a\right) a \cdot a \\
& =\left(\left(x^{3} y^{3} \cdot(a a \cdot x y)\right) a\right) a \cdot a \\
& =\left(\left(a a \cdot x^{4} y^{4}\right) a\right) a \cdot a=\left(\left(x^{4} y^{4} \cdot a\right) a \cdot a\right) a \cdot a \\
& =\left(\left(x^{4} y^{4} \cdot(a x \cdot a y)\right) a \cdot a\right) a \cdot a \\
& =\left(\left(a a \cdot x^{5} y^{5}\right) a \cdot a\right) a \cdot a=\left(\left(y^{5} x^{5} \cdot a a\right) a \cdot a\right) a \cdot a \\
& =\left(\left(a \cdot\left(y^{5} x^{5}\right) a\right) a \cdot a\right) a \cdot a \\
& =b a \cdot a \text {, where } b=\left(a \cdot\left(y^{5} x^{5}\right) a\right) a \cdot a \text {. }
\end{aligned}
$$

It is easy to see that $\max \left\{\left(f^{3} \circ g\right)(a), \gamma\right\} \geq \min \{(f \cap$ $g)(a), \delta\}$, which shows that $f^{3} \circ g \supseteq_{(\gamma, \delta)} f \cap g$ and similarly we can show that $g \circ f \supseteq_{(\gamma, \delta)} f \cap g$. Therefore $\left(f^{3} \circ g\right) \cap\left(g^{3} \circ f\right) \supseteq(\gamma, \delta) f \cap g$. It is easy to show that $\left(f^{3} \circ g\right) \cap\left(g^{3} \circ f\right) \subseteq_{(\gamma, \delta)} f \cap g$. Thus $f \cap g={ }_{(\gamma, \delta)}$ $\left(f^{3} \circ g\right) \cap\left(g^{3} \circ f\right)$.

$(v i) \Longrightarrow(v) \Longrightarrow(i v) \Rightarrow(i i i) \Longrightarrow(i i)$ are obvious cases.

$(i i) \Longrightarrow(i)$ : Let $f$ be any $\left(\epsilon_{\gamma}, \in_{\gamma} \vee q_{\delta}\right)$-fuzzy right ideal and $g$ be any $\left(\in_{\gamma}, \in_{\gamma} \vee q_{\delta}\right)$-fuzzy left ideal of $S$. Since $f \cap$ $g={ }_{(\gamma, \delta)}\left(f^{3} \circ g\right) \cap\left(g^{3} \circ f\right)$, therefore $f \cap g \subseteq_{(\gamma, \delta)} f^{3} \circ g$ and $f \cap g \subseteq_{(\gamma, \delta)} g^{3} \circ f$. Let $f \cap g \subseteq_{(\gamma, \delta)} f^{3} \circ g$. It is easy to show that $f^{3} \circ g \subseteq{ }_{(\gamma, \delta)} f \cap g$. Thus $f \cap g={ }_{(\gamma, \delta)} f^{3} \circ g$, and by using Corollary $8, S$ is $(2,2)$-regular. Similarly we can show that $S$ is $(2,2)$-regular if $f \cap g \subseteq(\gamma, \delta) g^{3} \circ f$.

Now let us explore the relationship between an $\left(\epsilon_{\gamma}, \epsilon_{\gamma}\right.$ $\vee q_{\delta}$ )-fuzzy idempotent subsets of an ordered $\mathcal{A}^{*} \mathcal{G}^{* *}$-groupoid $S$ and its $\left(\in_{\gamma}, \in_{\gamma} \vee q_{\delta}\right)$-fuzzy bi-ideals, explicitly, when will an $\left(\epsilon_{\gamma}, \in_{\gamma} \vee q_{\delta}\right)$-fuzzy idempotent subset of $S$ be an $\left(\epsilon_{\gamma}, \in_{\gamma}\right.$ $\vee q_{\delta}$ )-fuzzy bi-ideal. We answer this question in the following Proposition.

Proposition 1. Let $f$ be an $\left(\epsilon_{\gamma}, \in_{\gamma} \vee q_{\delta}\right)$-fuzzy idempotent subset of an ordered $\mathcal{A}^{*} \mathcal{G}^{* *}$-groupoid $S$, and let $f={ }_{(\gamma, \delta)} g \circ h$ for an $\left(\epsilon_{\gamma}, \in_{\gamma} \vee q_{\delta}\right)$-fuzzy left ideal $h$ and an $\left(\in_{\gamma}, \in_{\gamma} \vee q_{\delta}\right)$ fuzzy right ideal $g$ of $S$. Then $f$ is an $\left(\epsilon_{\gamma}, \in_{\gamma} \vee q_{\delta}\right)$-fuzzy bi-ideal of $S$.

Proof. By using Lemma 3, we have $(f \circ S) \circ f={ }_{(\gamma, \delta)}$ $(f \circ S) \circ(f \circ f) \subseteq_{(\gamma, \delta)}(g \circ S) \circ(S \circ h) \subseteq_{(\gamma, \delta)} g \circ h=_{(\gamma, \delta)} f$.

Another question is the realization of fuzzy subsets $f$ of an ordered $\mathcal{A}^{*} \mathcal{G}^{* *}$-groupoid which are both $\left(\epsilon_{\gamma}, \in_{\gamma} \vee q_{\delta}\right)$-fuzzy idempotent and $\left(\in_{\gamma}, \in_{\gamma} \vee q_{\delta}\right)$-fuzzy bi-ideal. This is given in 
the following Proposition.

Proposition 2. Let $f$ be an $\left(\epsilon_{\gamma}, \epsilon_{\gamma} \vee q_{\delta}\right)$-fuzzy idempotent subset and an $\left(\epsilon_{\gamma}, \epsilon_{\gamma} \vee q_{\delta}\right)$-fuzzy bi-ideal of an ordered $\mathcal{A}^{*} \mathcal{G}^{* *}$-groupoid $S$. Then there exist an $\left(\epsilon_{\gamma}, \in_{\gamma} \vee q_{\delta}\right)$-fuzzy left ideal $h$ and an $\left(\in_{\gamma}, \in_{\gamma} \vee q_{\delta}\right)$-fuzzy right ideal $g$ of $S$ such that $f={ }_{(\gamma, \delta)} g \circ h$.

Proof. Necessity. Setting $h={ }_{(\gamma, \delta)} S \circ f$ and $g={ }_{(\gamma, \delta)} S \circ f^{2}$, then by using Lemma 3, we have

$$
\begin{aligned}
g \circ h & ={ }_{(\gamma, \delta)}\left(S \circ f^{2}\right) \circ(S \circ f)={ }_{(\gamma, \delta)}\left(f^{2} \circ S\right) \circ(S \circ f) \\
& ={ }_{(\gamma, \delta)}((S \circ f) \circ(S \circ S)) \circ f^{2} \\
& ={ }_{(\gamma, \delta)}((S \circ S) \circ(f \circ S)) \circ f^{2} \\
& ={ }_{(\gamma, \delta)}(S \circ((f \circ f) \circ(S \circ S))) \circ f^{2} \\
& =(\gamma, \delta)(S \circ((S \circ S) \circ(f \circ f))) \circ f^{2} \\
& ={ }_{(\gamma, \delta)}(S \circ(f \circ(S \circ f))) \circ f^{2} \\
& ={ }_{(\gamma, \delta)}(f \circ(S \circ(S \circ f))) \circ f^{2} \\
& \subseteq{ }_{(\gamma, \delta)}(f \circ S) \circ f^{2} \subseteq(\gamma, \delta) f,
\end{aligned}
$$

and $f={ }_{(\gamma, \delta)} f^{2} \subseteq_{(\gamma, \delta)}\left(S \circ f^{2}\right) \circ(S \circ f)=_{(\gamma, \delta)} g \circ h$.

The Propositions 1 and 2 combined together give us a characterization Theorem which we state in the following

Theorem 10. Let $S$ be an ordered $\mathcal{A}^{*} \mathcal{G}^{* *}$-groupoid and $f$ be a an $\left(\epsilon_{\gamma}, \in_{\gamma} \vee q_{\delta}\right)$-fuzzy idempotent subset of $S$. Then $f$ is an $\left(\epsilon_{\gamma}, \in_{\gamma} \vee q_{\delta}\right)$-fuzzy bi-ideal of $S$ if and only if there exist an $\left(\epsilon_{\gamma}, \epsilon_{\gamma} \vee q_{\delta}\right)$-fuzzy left ideal $h$ and an $\left(\epsilon_{\gamma}, \in_{\gamma} \vee q_{\delta}\right)$-fuzzy right $g$ of $S$ such that $g \circ h={ }_{(\gamma, \delta)} f$.

\section{Conclusions and Applications}

The study of an algebraic structure using the order theory plays a prominent role in mathematics with wide ranging applications in many disciplines such as control engineering, computer arithmetics, coding theory, sequential machines and formal languages. In this regard, we have applied the order theory on the structure of an ordered $\mathcal{A G}$-groupoid and generalized the concept of an ordered semigroup using generated (generalized fuzzy) ideal theory.

We have considered the following problems in detail:

i) Compare $\left(\in_{\gamma}, \in_{\gamma} \vee q_{\delta}\right)$-fuzzy left/right ideals of an ordered $\mathcal{A} \mathcal{G}$-groupoid and respective examples are provided.

ii) Introduce the concept of an ordered $\mathcal{A}^{*} \mathcal{G}^{* *}$-groupoid (generalization of an ordered $\mathcal{A G}$-groupoid with left identity) and characterize it by using $\left(\epsilon_{\gamma}, \in_{\gamma} \vee q_{\delta}\right)$-fuzzy left/right ideals.

iii) Study the structural properties of an ordered $\mathcal{A}^{*} \mathcal{G}^{* *}$ groupoid in terms of its semilattices, $(2,2)$-regular class and generated commutative monoids.

This paper generalized the theory of an $\mathcal{A G}$-groupoid in the following ways:

$i)$ In an $\mathcal{A G}$-groupoid (without order) by using the $\left(\epsilon_{\gamma}, \in_{\gamma}\right.$ $\left.\vee q_{\delta}\right)$-fuzzy ideals. ii) In an $\mathcal{A} \mathcal{G}$-groupoid (with and without order) by using fuzzy ideals instead of $\left(\in_{\gamma}, \in_{\gamma} \vee q_{\delta}\right)$-fuzzy ideals.

Some important issues for future work are:

i) To develop strategies for obtaining more valuable results in related areas.

ii) To apply these notions and results for studying $\left(\in_{\gamma}\right.$ ,$\left.\in_{\gamma} \vee q_{\delta}\right)$-fuzzy ideals in $\mathcal{L} \mathcal{A}$-semihypergroups and soft $\mathcal{L} \mathcal{A}$ semigroups.

Finally this paper will give us the extension of the work carried out in [22] in a more generalized substructure of an ordered $\mathcal{A G}$-groupoid.

\section{Acknowledgements}

The author gratefully acknowledged the financial support from the Deanship of Scientific Research and Graduate studies at Amman Arab University in Jordan.

\section{REFERENCES}

[1] M. A. Kazim and M. Naseeruddin, On almost semigroups, The Aligarh Bulletin Of Mathematics, Vol. 2, 1-7, 1972.

[2] P. V. Protić and N. Stevanović. AG-test and some general properties of Abel-Grassmann's groupoids, Pure Mathematics and Applications, Vol. 6, No.4 , 371-383, 1995.

[3] Q. Mushtaq and S. M. Yusuf, On LA-semigroups, The Aligarh Bulletin Of Mathematics, Vol. 8, 65-70, 1978.

[4] W. A. Dudek and R. S. Gigon. Congruences on completely inverse $\mathrm{AG}^{* *}$-groupoids, Quasigroups and related systems, Vol. 20, 203-209, 2012.

[5] W. A. Dudek and R. S. Gigon. Completely inverse AG**groupoids. In Semigroup Forum, Vol. 87, No.1 , 201-229, 2013.

[6] M. Khan. Some studies in $\mathrm{AG}^{*}$-groupoids, $\mathrm{Ph}$. D Thesis, Quaidi-Azam University, Pakistan, 2008.

[7] Q. Mushtaq and S. M. Yusuf. On locally associative left almost semigroups, J. Nat. Sci. Math., Vol. 19, No.1 , 57-62, 1979.

[8] N. Stevanović and P. V. Protić. Composition of AbelGrassmann's 3-bands, Novi Sad, J. Math., Vol. 34, No.2 , 175$182,2004$.

[9] L. A.Zadeh. Fuzzy sets, Information and Control, Vol. 8, No.3, 338-353, 1965.

[10] Y. Al-Qudah and N. Hassan. Operations on complex multifuzzy sets, Journal of Intelligent and Fuzzy Systems, Vol.33, No.3, 1527-1540, 2017.

[11] Y. Al-Qudah and N. Hassan. Complex Multi-Fuzzy Relation for Decision Making using Uncertain Periodic Data, International Journal of Engineering and Technology (UAE), Vol.7, No.4, 2437-2445, 2018.

[12] Y. Al-Qudah and N. Hassan. Complex multi-fuzzy soft set: Its entropy and similarity measure, IEEE Access, Vol.6, No.3, 65002-65017, 2018. 
[13] V. Murali, Fuzzy points of equivalent fuzzy subsets, Information Sciences, Vol.815, 277-288, 2004.

[14] P.M. Pu and Y.M. Liu. Fuzzy topology I, neighborhood structure of a fuzzy point and Moore Smith convergence, Journal of Mathematical Analysis and Applications, Vol.76, No.2, 571$599,1980$.

[15] S. K Bhakat and P.Das. On the definition of a fuzzy subgroups, Fuzzy sets and Systems, Vol.51, No.2, 235-241, 1992.

[16] A. Rosenfeld. Fuzzy groups, Journal of mathematical analysis and applications, Vol.35, No.3, 512-517, 1971.

[17] B. Davvaz. $(\in, \in \vee q)$-fuzzy subnear-rings and ideals, Soft Computing, Vol.10, No.31, 206-211, 2006.

[18] O. Kazanci and S. Yamak. Generalized fuzzy bi-ideals of semigroup, Soft computing, Vol.12, No.11, 1119-1124, 2008.
[19] M. Shabir, Y. B. Junan Y. Nawaz. Characterizations of regular semigroups by $(\alpha, \beta)$-fuzzy ideals, Computers and Mathematics with Applications, Vol.59, No.1, 161-175, 2010.

[20] M. Shabir, Y. B. Junan Y. Nawaz, Semigroups charactarized by $\left(\in, \in \vee q_{k}\right)$-fuzzy ideals, Computer and Mathematics with applications, Vol.60, No.5,1473-1493, 2010.

[21] F. Yousafzai, A. Khan, V. Amjid and A. Zeb. On fuzzy fully regular ordered AG-groupoids, Journal of Intelligent and Fuzzy Systems, Vol.26, No.6, 2973-2982, 2014.

[22] F. Yousafzai, N. Yaqoob and A. Zeb. On generalized fuzzy ideals of ordered AG-groupoids, International Journal of Machine Learning and Cybernetics, Vol.7, No.6, 995-1004, 2016. 